\title{
Transcriptomic characterization of the immunogenetic repertoires of heteromyid rodents
}

\author{
Nicholas J Marra ${ }^{1 *}$ and J Andrew DeWoody ${ }^{2,3}$
}

\begin{abstract}
Background: When populations evolve under disparate environmental conditions, they experience different selective pressures that shape patterns of sequence evolution and gene expression. These may be manifested in genetic and phenotypic differences such as a diverse immunogenetic repertoire in species from tropical latitudes that have greater and/or different parasite burdens than more temperate species. To test this idea, we compared the transcriptomes of one tropical species (Heteromys desmarestianus) and two species from temperate latitudes (Dipodomys spectabilis and Chaetodipus baileyi) from the Heteromyidae. We did so in a search for positive selection on sequences and/or differential expression, while controlling for phylogenetic history in our choice of species.

Results: We identified 127,812 contigs and annotated 34,878 of these, identifying immune genes associated with interleukins, cytokines, and the production of mast cells. We identified 632 genes that were upregulated in $H$. desmarestianus (8.7\% of genes tested) and 492 (6.7\%) that were downregulated. Gene ontology terms including "immune response" were associated with 31 (4.9\%) of the 632 upregulated genes. We found preliminary evidence for positive selection on three genes (Palmitoyltransferase ZDHHC5 Ubiquitin-conjugating enzyme E2 N, Krueppel-like factor 10, and Spindle and kinetochore-associated protein 1) along the H. desmarestianus lineage.

Conclusions: Overall our findings pinpoint genes in species from disparate environments that are on different evolutionary trajectories in terms of expression levels and/or nucleotide sequence. Our data indicate there are significant differences in the expression of genes among the spleen transcriptomes of these species and that a number of these differentially expressed genes do not show the same pattern of differential expression in another tissue type. This points to the possibility of expression differences between these species specific to the spleen transcriptome.
\end{abstract}

Keywords: Transcriptomics, Spleen, Latitudinal diversity gradient, Differential gene expression, Evolutionary genomics

\section{Background}

Variation in natural selection between habitats can lead to the evolution of phenotypic differences between related taxa. For example, multiple studies have shown that freshwater populations of threespine stickleback, Gasterosteus aculeatus, consistently exhibit loss of body plate armor that is found in marine populations of the species and that there is evidence of a common genetic basis for these alternate phenotypes [1,2]. Studies of environmental contrasts can provide an extreme but useful framework for identifying the impact of disparate selective

\footnotetext{
* Correspondence: njm77@cornell.edu

'Department of Population Medicine and Diagnostic Sciences, Cornell University, S3-111 Schurman Hall, Tower Road, Ithaca, NY 14853, USA Full list of author information is available at the end of the article
}

pressures on the genome. For instance, the latitudinal diversity gradient (LDG), which describes the increase in species richness that occurs from the poles to the tropics, may be due to unstable climates and abiotic factors that constrain evolution in temperate latitudes or to the increased biotic interactions that drive diversity in tropical locales [3,4]. For example, host-parasite interactions may act as powerful selective agents in tropical locales where overall species diversity is high.

Parasites (and predators) are known to impose greater selective pressure on their hosts in tropical environments than in temperate environments $[5,6]$. This could be due in part to year round conditions conducive to high host productivity which supports high parasite diversity and abundance [7]. If selection due to parasite load is greatest 
in the tropics, or varies relative to temperate areas, tropical and temperate hosts should differ with respect to measures such as host infection status, immune response, and parasite abundance.

Several empirical studies have evaluated parasite abundance in temperate and tropical birds and found evidence of increased immune response and/or parasite richness at lower, tropical latitudes [8-10] although this directionality is not universal $[11,12]$. Similarly, greater parasite load in tropical populations of the lizard Eulamprus quoyii has been reported [13]. In primates, a literature survey of pathogens and host ranges found evidence of the LDG for protozoan parasites but not for viruses [14], and within humans there is evidence of the LDG for several human parasites [15]. Finally, species richness in African ticks is correlated with the LDG as measured by their avian and mammalian hosts [16].

Evidence for immunogenetic divergence along an LDG exists as well. For instance, a study of genome-wide genetic differentiation (measured by $F_{s t}$ ) between tropical and temperate populations of Drosophila melanogaster found multiple immunity related genes that were significantly differentiated between the populations [17]. The same study also found enrichment for innate immunity genes involved in the Toll signaling pathway in the $F_{\mathrm{ST}}$ outliers indicative of possible selection on those loci. Another example is the pattern of increased MHC diversity in Atlantic salmon populations at lower latitudes and the fact that bacterial diversity is greater in rivers at lower latitudes, suggesting a possible link between increased pathogen abundance and genetic variation [18].

Whereas those earlier studies sought to quantify proximate selective pressures, we aimed to determine if selective pressures shaped sequence evolution and/or gross changes in gene expression of immune genes in a tropical rodent compared to temperate relatives. We used as evolutionary models the forest spiny pocket mouse (Heteromys desmarestianus) and two temperate relatives, Chaetodipus baileyi (Bailey's pocket mouse) and Dipodomys spectabilis (banner-tailed kangaroo rat).

All three of our study species are members of the Heteromyidae, a family of new world rodents whose native range extends from North America through Central America and into northern South America [19,20] (see Figure 1). This family is comprised of three subfamilies which diverged from one another roughly 22 mya [21]: the Heteromyinae, Perognathinae, and the Dipodomyinae (represented in our study by $H$. desmarestianus, $C$. baileyi, and D. spectabilis, respectively). Heteromys spp. have the most tropical latitudinal distribution of the family and are often found in lowland rainforest whereas Chaetodipus spp. and Dipodomys spp. generally have temperate desert distributions $[19,20,22]$. The historical biogeography of the species used in this study has not been described in detail, but Miocene fossils of the Perognathinae and Dipodomyinae ancestors have been found in the southwestern US and northern Mexico. These regions developed into arid deserts during the Pleistocene (historical biogeography of the Heteromyidae reviewed in [20]). No fossil record exists for Heteromys in Central America, but members of the genus are thought to have inhabited Central America prior to expanding into portions of northern South America as early as 3 mya [20,23]. This history points to the long-term presence of these species in temperate and tropical latitudes, respectively.

Heteromyid rodents are afflicted by a variety of endoand ectoparasites including viruses, bacteria, protozoa, nematodes, mites, ticks, lice, and other parasites [20]. Apparent parasite burdens increase as a species is more intensively studied [20] and this ascertainment bias is difficult to ameliorate. However, there are cases where our tropical study species $(H$. desmarestianus) appears to have a greater parasite diversity than its more temperate relatives. For example, sucking lice (Fahrenholzia species) are known to parasitize members of the Heteromyidae where a few individuals feed on the blood of a single host [26]. H. desmarestianus is one of the few host species in this complex that is parasitized by multiple sucking lice species [20,26]. Overall, we expect that rodent species from more temperate latitudes have been infected with a different suite of parasites than the more tropical rodent species (i.e., $H$. desmarestianus).

To identify whether tropical species display molecular signatures of divergent selective pressure on immune response, we used a phylogenetic framework to compare these three rodent species. Our study framework provides a phylogenetic control due to our species selection. The Perognathinae and Heteromyinae are more closely related to each other than either are to the Dipodomyinae $[19,21]$. Thus, we chose to compare a tropical species from the Heteromyinae to two species from temperate latitudes that were members of the Perognathinae and Dipodomyinae. These two species co-occur at our temperate latitude collection site and have similar ranges (Figure 1). By focusing on differences that were consistent between the tropical species and each of the temperate latitude species, any differences in gene expression and sequence evolution are more likely due to consistent life history differences between the two groups rather than phylogenetic divergence.

Our three study species have been the subject of a variety of ecological $[27,28]$, behavioral $[29,30]$, physiological [31-34], and phylogenetic studies [19,21,26]. Nevertheless, genomic resources for the group are limited and we have conducted transcriptome sequencing to expand the resources available to study various aspects of heteromyid biology. Previously, we sought to characterize the genetic basis of the superior osmoregulation and kidney function of D. spectabilis and C. baileyi [35]. There, we conducted 


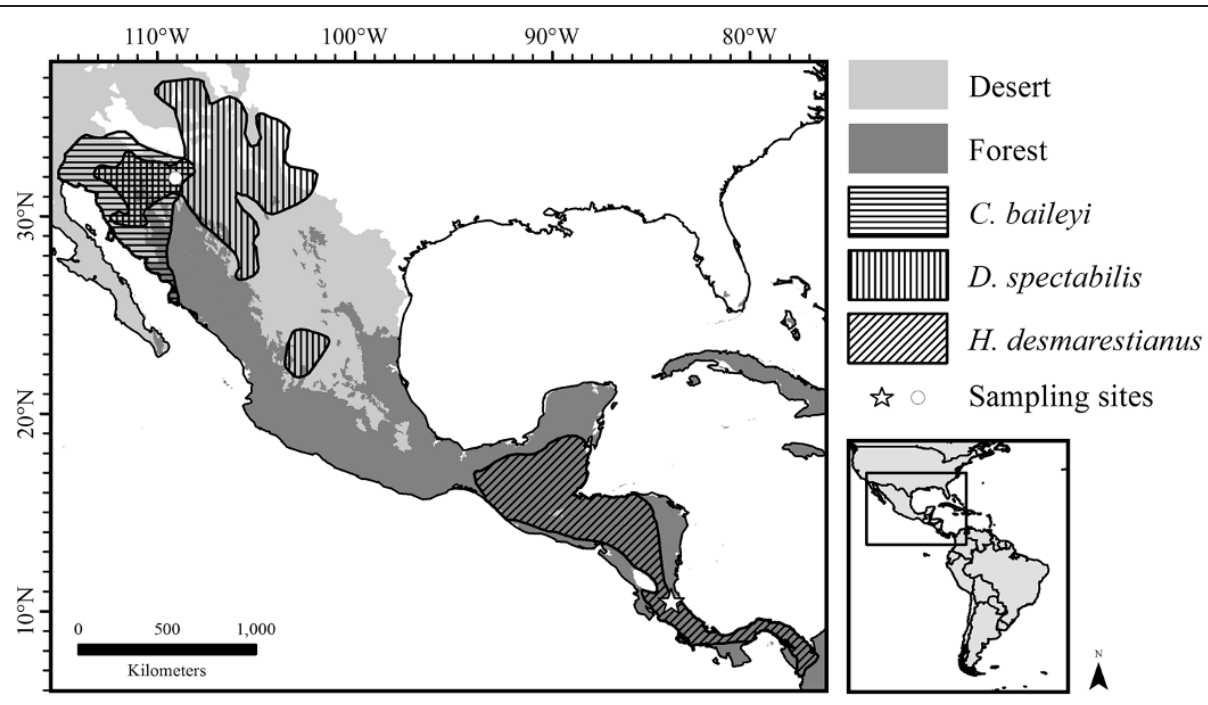

Figure 1 Distribution and sampling locations of Chaetodipus baileyi, Dipodomys spectabilis, and Heteromys desmarestianus. Range map of North America displaying habitat and latitude for all three study species constructed on Arc-GIS. Areas occupied by C. baileyi are highlighted by horizontal lines, areas occupied by $D$. spectabilis are denoted by vertical lines, and the range of $H$. desmarestianus is denoted by diagonal lines. Overlap between C. baileyi and D. spectabilis is cross hatched. The white star denotes the sampling location of $H$. desmarestianus at La Selva Biological Station, Costa Rica whereas the white circle marks the sampling location for C. baileyi and D. spectabilis at Portal, AZ, USA, Species range data were obtained from IUCN Red List of Threatened Species. Version 2013.1. [24]. Habitat data is from [25].

RNA-seq of kidney tissue to identify consistent patterns of expression and selection in the kidney transcriptome of these two desert species relative to $H$. desmarestianus [35]. Here, we focus not on osmoregulation in the heteromyid kidney but on immunogenetic profiles of the heteromyid spleen. We do so in an effort to examine how selective pressures may drive transcriptomic differences between contrasting environments while simultaneously providing immunogenetic resources across the Heteromyidae.

This research forms a significant first step in identifying the presence and/or effects of a drastically different selective pressure on the tropical $H$. desmarestianus relative to the temperate $D$. spectabilis and C. baileyi, which are both constrained by adaptation to desert habitat $[19,35]$. We used spleen tissue because its relative size has been used previously as a proxy for investment in immune defense [9] and because it has been used to characterize the major histocompatibility complex (MHC genes) in D. spectabilis [36]. The rodent spleen also functions in filtering and defense against blood borne pathogens (e.g. malarial parasites; reviewed in [37]) and the spleen has been implicated in both innate and adaptive immune response (reviewed in [38-40]). There is also evidence that gene expression in the spleen can be affected by the action of external pests/parasites feeding on hosts. For example, studies have shown that extracts from the salivary glands of sand and black flies can affect cell proliferation and gene expression in mouse spleen cells $[41,42]$. Thus, we expect that gene expression in the spleen is sensitive to the variable immune challenges represented by different parasite assemblages on our species and that over time their spleen transcriptomes have been shaped by these different pressures.

\section{Methods}

\section{Sample collection}

The samples for this project were collected from the field as described in [43] and [35]. Briefly, spleen tissue from each of four adult $D$. spectabilis (2 males and 2 females) and four adult $C$. baileyi (2 males and 2 females) was harvested during collection trips to the same locale near Portal, AZ (about 31 $37^{\circ} \mathrm{N}$ latitude) in December 2009 and December 2011, respectively. Additionally, spleen tissue was collected from four individual $H$. desmarestianus (1 male and 3 females) trapped within La Selva Biological Station in the lowlands of northern Costa Rica (roughly $10^{\circ} 25^{\prime} \mathrm{N}$ latitude) in June 2010. All 12 individuals were euthanized according to approved Purdue Animal Care and Use protocols, and tissue was harvested via dissections. Tissue was minced immediately and frozen in TRIzol $^{\odot}$ reagent (Invitrogen) prior to transport back to Purdue University.

\section{RNA preparation and sequencing}

At Purdue University, total RNA was extracted from a representative portion of each sample using $\mathrm{TRIzol}^{\circ}$ reagent (Invitrogen) according to manufacturer instructions. For the D. spectabilis and $H$. desmarestianus samples, $1 / 2$ plate of 454 sequencing was conducted using cDNA synthesized from our initial RNA extractions with the ClonTech SMART cDNA synthesis kit [44] with a modified 
CDS III/3' primer (see $[43,45,46])$. For Illumina sequencing, fresh RNA extractions were used to obtain total RNA for all 12 samples, which were then purified using RNA Clean and Concentrator columns (Zymo Research). RNA quality was evaluated with an Agilent 2100 Bioanalyzer before a uniquely barcoded $2 \times 100$ paired end library was constructed from each sample. All 12 libraries were then pooled and sequenced in two separate lanes of sequencing using an Illumina HiSeq 2000. This sequencing effort also included 12 libraries for a separate study [35], in total the same 24 libraries (12 of which were for this study) were sequenced in each lane of Illumina sequencing.

\section{Transcriptome assembly}

Our assembly methodology (Table 1 lists programs and versions used here) closely follows our previous work on kidney transcriptomes [35]. Adaptors and library synthesis primers were trimmed from sequences and the libraries were then filtered to remove poor quality reads. DeconSeq version 0.4.1 [47] was used to remove reads with a minimum overlap of $60 \%$ and $>80 \%$ similarity to the rRNA sequence. The filtered reads from all four individuals per species were pooled to assemble a speciesspecific de novo spleen reference transcriptome $(\mathrm{n}=3$ de novo assemblies) by first assembling all Illumina reads with the Trinity assembler [48]. Then Trinity contigs were fragmented into $\leq 1 \mathrm{~kb}$ pieces with a script from http://www.clarkfrancis.com/blast/fragblast.html before assembly with 454 reads in gsAssembler. The contigs from this gsAssembler assembly constituted our de novo transcriptome for each species.

Table 1 List of programs used throughout the paper with a brief description of their function

\begin{tabular}{|c|c|c|c|}
\hline Program & Version & Use & Citation \\
\hline DeconSeq & 0.4 .1 & $\begin{array}{l}\text { Read filtering and } \\
\text { processing }\end{array}$ & {$[47]$} \\
\hline Trinity & $\begin{array}{l}\text { trinityrnaseq- } \\
\text { r2013-02-25 }\end{array}$ & $\begin{array}{l}\text { de novo assembly } \\
\text { of Illumina reads }\end{array}$ & {$[48]$} \\
\hline gsAssembler & 2.6 & $\begin{array}{l}\text { de novo assembly of } \\
\text { Illumina and } 454 \text { reads }\end{array}$ & $\begin{array}{l}\text { Roche/454 } \\
\text { manual }\end{array}$ \\
\hline Bowtie & 1.0 .0 & $\begin{array}{l}\text { Paired end read mapping } \\
\text { implemented in RSEM }\end{array}$ & {$[49]$} \\
\hline RSEM & 1.2 .0 & $\begin{array}{l}\text { Uses bowtie and maximum } \\
\text { likelihood approaches to } \\
\text { map reads to a scaffold } \\
\text { and give the number of } \\
\text { reads that map to a } \\
\text { sequence }\end{array}$ & {$[50]$} \\
\hline DESeq & 1.14 & $\begin{array}{l}\text { Uses read counts to test } \\
\text { for differential expression }\end{array}$ & {$[51]$} \\
\hline MUSCLE & 3.6 & Multiple sequence alignment & {$[52]$} \\
\hline PAML & 4.7 & $\begin{array}{l}\text { Analysis of protein and DNA } \\
\text { sequences using maximum } \\
\text { likelihood based methods }\end{array}$ & {$[53,54]$} \\
\hline
\end{tabular}

\section{BLAST annotation and GO enrichment}

After filtering out all contigs that were $\leq 100 \mathrm{bp}$, we annotated the three resulting de novo transcriptomes with BLASTx [55] using the Swiss-Prot database and an evalue threshold of e $\leq 1 \times 10^{-6}$ [46] to obtain annotated transcriptomes from the pool of four individuals per species. Using Blast2GO [56], we obtained gene ontology (GO) terms for our annotated contigs and conducted a Fisher's exact test to identify gene ontologies that were consistently overrepresented or underrepresented in the $H$. desmarestianus dataset at $\mathrm{p} \leq 0.05$ relative to the other two species.

\section{Test for Differential Expression (DE)}

As in [35], we obtained measures of gene expression for each of four individuals per species by mapping the individual Illumina reads back to our reference de novo transcriptomes that had been assembled in gsAssembler. Mapping was conducted with Bowtie [49] as run within the program RSEM [50]. We did so using the default parameters in the program RSEM [50]. RSEM allows a maximum of 2 mismatches per 25 bp seed but has Bowtie report all possible mapping locations for each read (reads that do not map are discarded). RSEM then uses this information in an algorithm to calculate the maximum likelihood estimate of the number of fragments assigned to each contig and thus account for reads that map to multiple possible locations $[50,57]$. We rounded this value to the nearest whole number to assign a read count for individual contigs.

In principle, some genes could be covered by more than one contig due to several factors such as alternative splicing or incomplete coverage (gaps). Thus we summed read counts for all contigs with identical annotations to get a single read count for each gene. If ten or more reads from three of the four individuals mapped back to a gene then it was retained for DE analysis (otherwise it was discarded). Differential expression was evaluated between the three species by conducting the three possible pairwise analyses (C. baileyi vs. $H$. desmarestianus; $D$. spectabilis vs. $H$. desmarestianus; and C. baileyi vs. D. spectabilis) using the $\mathrm{R}$ package DESeq [51]. This program controls for library size, models the count data with a negative binomial distribution, and identifies significantly DE genes after a Benjamini-Hochberg correction for multiple testing and a threshold of an adjusted $\mathrm{p}\left(\mathrm{p}_{\mathrm{adj}}\right)<0.05[51,58]$. Default parameters were used as described in the program's documentation.

To gain greater insights into the gene transcripts, we tested for the enrichment of GO terms. First, we employed Fisher's exact test to identify the GO terms that were enriched in genes overexpressed in $H$. desmarestianus relative to the two temperate species. Then, we performed another Fisher's exact test to identify the GO 
terms that were enriched in genes underexpressed in $H$. desmarestianus relative to the two temperate species.

\section{Test for positive selection}

Sequences were treated in a similar manner to [35] to build alignments of putative orthologues for four species (M. musculus, C. baileyi, D. spectabilis, and $H$. desmarestianus). After we obtained the best reciprocal BLAST hits for contigs from D. spectabilis, C. baileyi, and H. desmarestianus, we constructed sets of putative orthologues composed of one sequence from each species (three sequences per set of orthologues). We required all putative orthologues to have the same hit to known Mus musculus protein sequences (obtained from Ensembl release 75) during a BLASTx search with a cutoff of e-value $<1 \times 10^{-9}$. We further required that all species had a full-length alignment with the M. musculus sequence according to the BLASTx analysis. The coordinates of the full-length BLASTx search were used to trim each sequence of the orthologue set to the 5' and 3' positions of the alignment with $M$. musculus. We included the $M$. musculus coding sequence of the $M$. musculus protein hit as the fourth and final sequence for the set of orthologues.

All sets of four orthologous nucleotide sequences that remained after this filtering were aligned using MUSCLE $\mathrm{v}$. 3.6 [52] in the first forward reading frame of the $M$. musculus coding sequence. These alignments were used in the codeml program in PAML $4.7[53,54]$ to test for selection along the tropical $H$. desmarestianus lineage. A guide tree was constructed from cytochrome $c$ oxidase subunit I (COI) sequences from the four species; the three heteromyid COI sequences were from [21] (GenBank accession numbers: EF156845.1, EF156850.1, EF156856.1); Mus musculus COI sequence was from the M. musculus mitochondrial genome, GenBank accession: NC_005089.1). We allowed the branch lengths of the tree to be estimated by codeml for each alignment separately and we employed the clean data option to remove sites where gaps were encountered.

We used two tests to explicitly test whether the branch leading to the tropical species, $H$. desmarestianus, exhibited evidence of positive selection as indicated by an elevated ratio of nonsynonymous to synonymous changes (a $\mathrm{dn} / \mathrm{ds}$ or $\omega>1$ ). For each test we evaluated a model where $\omega$ was allowed to vary on a specified lineage vs. a null model where $\omega$ was the same for all lineages. First we conducted the 'branch' test $[53,54,59,60]$ where model 2 (the alternative model) is compared to model 0 (null model). In this case, two values of $\omega$ are estimated for the alternative model (one for a marked branch and one for the remaining lineages) and one value of $\omega$ is estimated for the entire tree in the null model. For our second comparison we allowed $\omega$ to vary across both lineages and sites. In this test we used model 2 as the alternative model with the additional modification of NSsites $=2$ and compared this to a null model of model 2 and NSsites $=2$ but where we fixed $\omega$ at a value of 1 for site class 2 .

For a detailed explanation, see [61,62] but briefly: under NSsites $=2$ there are three possible classes for a site (site-classes 0,1 , and 2) and there are two branch types (background and foreground, the latter being our marked branch, $H$. desmarestianus). Each site class has its own value of $\omega$ that is estimated. In the null model, the value of site class 0 is $0 \leq \omega \leq 1$ for both branch types, the value of site class 1 is $\omega=1$ for both branch types, and the value of site class 2 is $\omega=1$ for the background branches and is fixed to be $\omega=1$ for the foreground branches as well. The alternative model is the same as the null model except that for site class $2 \omega \geq 1$ is allowed for the foreground branch. This second comparison corresponds to the 'branch-site' models and we refer to it as the branch-sites test $[61,62]$.

These tests were conducted in a similar manner to those of [35] but here we tested for positive selection on the branch leading to the tropical species, $H$. desmarestianus. Positive selection was indicated by $\omega>1$ and a significant difference between the tested and null models was indicated by a Likelihood Ratio Test (LRT). To assess significance we obtained a p-value for each LRT from the chi-squared distribution and then adjusted for multiple testing by applying a Benjamini-Hochberg correction for false discovery rate using the p.adjust function in R. Genes were identified as significant if they had an adjusted $\mathrm{p}$-value $\left(\mathrm{p}_{\text {adj }}\right)<0.05$.

\section{Results}

Sequencing, assembly, and annotation

The sequences generated for this paper have been deposited in the NCBI-short read archive (NCBI SRA: Reads for NCBI BioProject: PRJNA261897, PRJNA261898, and PRJNA261902) and final transcriptome assemblies are available at Dryad http://dx.doi.org/10.5061/dryad.qn474. From the 2 lanes of Illumina sequencing we obtained $147,565,714$ paired-end reads $(2 \times 100$ bp reads $)$ across all species. For the 454 pyrosequencing of $H$. desmarestianus and D. spectabilis, we obtained an additional 322,763 reads (mean length 294 bp; see Table 2). Mean contig length was roughly $1 \mathrm{~kb}$ for all three species (Table 3). With the GO term annotations from Blast2GO [56] we conducted a Fisher's exact test to test for enrichment of GO terms in the $H$. desmarestianus dataset. There were $97 \mathrm{GO}$ terms that were either enriched or underrepresented in the $H$. desmarestianus dataset, meaning there were either proportionally more or proportionally less genes (respectively) in the $H$. desmarestianus dataset with those GO terms than in the combined $D$. spectabilis and C. baileyi datasets. Of these, $73 \mathrm{GO}$ terms were overrepresented and 24 were underrepresented in $H$. 
Table 2 Descriptive statistics from combined 454 and Illumina DNA sequencing runs of three heteroymid rodents

\begin{tabular}{llll}
\hline & $\begin{array}{l}\text { Dipodomys } \\
\text { spectabilis }\end{array}$ & $\begin{array}{l}\text { Heteromys } \\
\text { desmarestianus }\end{array}$ & $\begin{array}{l}\text { Chaetodipus } \\
\text { baileyi }\end{array}$ \\
\hline 454 reads & 203,096 & 119,667 & N/A \\
mean 454 read length & $292 \mathrm{bp}$ & $299 \mathrm{bp}$ & $\mathrm{N} / \mathrm{A}$ \\
Illumina reads & $57,505,313$ & $46,290,213$ & $43,770,188$ \\
\hline
\end{tabular}

desmarestianus (see Additional file 1: Table S1). Several of these GO terms are closely linked to immunity including GO:0030368: interleukin-17 receptor activity; GO:0002215: defense response to nematode; GO:1900017: positive regulation of cytokine production involved in inflammatory response; and GO:0043306: positive regulation of mast cell degranulation. Although these terms are no longer significant after FDR correction, their raw $\mathrm{p}<0.05$ and more importantly their link to immunity indicate that the genes they describe may warrant further investigation.

\section{Differential gene expression}

From the DESeq analysis, out of 7,266 genes that passed the filtering step for the differential expression (DE) test, we identified 2,786, 2,533, and 2,893 significantly DE genes in the D. spectabilis vs. $H$. desmarestianus, C. baileyi vs. $H$. desmarestianus, and C. baileyi vs. D. spectabilis comparisons, respectively. The comparisons of evolutionary interest are the environmental contrasts whereby a temperate species (C. baileyi or D. spectabilis) is compared to the tropical species ( $H$. desmarestianus). There were 1,274 genes that were DE in both of these comparisons. Of these, 49.6\% (632 genes) were consistently upregulated (had higher expression) in $H$. desmarestianus relative to the temperate species and $38.6 \%$ (492 genes) were consistently downregulated, whereas $11.8 \%$ (150 genes) showed an inconsistent pattern of expression (upregulated in one comparison, downregulated in the other) (see Figure 2).

When applying a FDR correction (at a threshold of 0.05), the 632 genes that were consistently upregulated had a statistically significant overrepresentation of $78 \mathrm{GO}$ terms and an underrepresentation of $48 \mathrm{GO}$ terms. Table 4 contains

Table 3 Summary of combined 454/Illumina assembly and annotation for contigs

\begin{tabular}{llll}
\hline & $\begin{array}{l}\text { Dipodomys } \\
\text { spectabilis }\end{array}$ & $\begin{array}{l}\text { Heteromys } \\
\text { desmarestianus }\end{array}$ & $\begin{array}{l}\text { Chaetodipus } \\
\text { baileyi }\end{array}$ \\
\hline \# of contigs & 58,435 & 35,318 & 34,059 \\
Mean contig length & $888 \mathrm{bp}$ & $1,158 \mathrm{bp}$ & $1,251 \mathrm{bp}$ \\
$\begin{array}{l}\text { Number of gene } \\
\text { annotations }\end{array}$ & 12,160 & 11,526 & 11,192 \\
$\begin{array}{l}\text { Annotated genes } \\
\text { unique to the species }\end{array}$ & 1,045 & 1,618 & 602 \\
\hline
\end{tabular}

the GO terms that were overrerpresented in the set of upregulated genes after filtering for the most specific GO level. This filtering removes higher order, less specific terms within a hierarchy of GO terms if all terms in the lineage of terms are enriched. For example, if a term such as 'cell adhesion' and the more specific term 'positive regulation of cell adhesion' were both enriched, then the filtering employed for Table 4 would remove the term 'cell adhesion' from the output. When evaluating the 492 genes that were consistently downregulated in $H$. desmarestianus, no terms were significantly overrerpresented or underrepresented.

\section{Selection tests}

After filtering, there were 395 MUSCLE alignments of orthologous sequences as determined by our reciprocal BLAST analysis. The PAML analysis of this 395 gene dataset yielded 45 genes where model 2 varied from model $0(\mathrm{p}<0.05, \mathrm{df}=1)$ for the branch test. Of these 45 genes, 4 were significant $\left(\mathrm{p}_{\mathrm{adj}}<0.05, \mathrm{df}=1\right)$ in the branch test after correction for multiple testing. This indicates that for these genes a model where the tropical lineage had a different $\omega$ from the rest of the tree was able to fit the data better than when one $\omega$ was applied to the whole tree. However, none of these 4 genes demonstrated an elevated $\omega>1$ under model 2 and thus these 4 genes lacked evidence for positive selection.

The branch-sites test identified 8 genes with a substantial difference between the model which allowed selection and the null model $(\mathrm{p}<0.05, \mathrm{df}=1)$. The test remained significant for one gene (Swiss-Prot symbol: $\operatorname{lrc} 8 a$, gene symbol: $\operatorname{Lrrc} 8 a$ ) after correction for multiple testing $\left(\mathrm{p}_{\mathrm{adj}}<0.05, \mathrm{df}=1\right.$ ), but this gene did not display an elevated $\omega$ indicative of positive selection. Of the other 7 genes that exhibited a preliminary difference in the branch-sites test, 6 had an $\omega>1$ along the $H$. desmarestianus branch. We checked these 6 alignments by eye and discovered alignment errors in 3 of them. Thus, 3 genes remained that displayed an initial difference in the branch-sites test and exhibited elevated $\mathrm{dn} / \mathrm{ds}$ ratios, indicating preliminary evidence for positive selection. However these 3 genes failed to remain significant following correction for multiple testing (Table 5).

\section{Discussion}

The role of selection in driving and shaping genetic variation has long been a subject of considerable interest in evolutionary biology. Some of the best molecular examples of natural selection come from the study of genes that play a role in immunity to infectious disease and/or pathogens $[63,64]$. Indeed, a recent review found that 84 genes related to immunity exhibited interspecific signs of strong selection between the human and chimpanzee lineages [63]. 


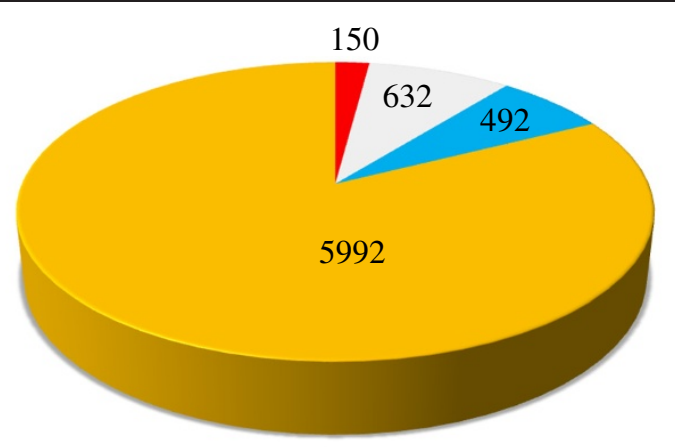

\section{$\square$ Inconsistent $\square$ Upregulated $\square$ Downregulated $\square$ Not DE}

Figure 2 Breakdown of genes tested for differential expression between tropical and temperate heteromyid rodents. Tests for differential expression (DE) between the tropical species Heteromys desmarestianus and two related temperate species, Dipodomys spectabilis and Chaetodipus baileyi. 7,266 genes were tested for DE and 1,274 were significantly DE in both of these comparisons; significance was identified after a Benjamini-Hochberg correction.

Table 4 GO terms significantly enriched in the set of genes that are upregulated in Heteromys desmarestianus

\begin{tabular}{|c|c|c|c|c|}
\hline GO-ID & Term & Category & P-Value & BH FDR \\
\hline GO:0030041 & Actin filament polymerization & $P$ & $3.90 \mathrm{E}-04$ & $4.58 \mathrm{E}-02$ \\
\hline GO:0046165 & Alcohol biosynthetic process & $P$ & $1.39 \mathrm{E}-04$ & 2.20E-02 \\
\hline GO:0030154 & Cell differentiation & $P$ & 3.66E-04 & $4.48 \mathrm{E}-02$ \\
\hline GO:0034329 & Cell junction assembly & $P$ & $3.29 \mathrm{E}-04$ & 4.10E-02 \\
\hline GO:0032989 & Cellular component morphogenesis & $P$ & $3.72 \mathrm{E}-04$ & 4.51E-02 \\
\hline GO:0030198 & Extracellular matrix organization & $P$ & $3.98 \mathrm{E}-04$ & $4.58 \mathrm{E}-02$ \\
\hline GO:0006775 & Fat-soluble vitamin metabolic process & $P$ & 2.57E-04 & $3.32 \mathrm{E}-02$ \\
\hline GO:0008610 & Lipid biosynthetic process & $P$ & 4.11E-04 & 4.65E-02 \\
\hline GO:0045785 & Positive regulation of cell adhesion & $P$ & $1.55 \mathrm{E}-04$ & $2.30 \mathrm{E}-02$ \\
\hline GO:0030335 & Positive regulation of cell migration & $P$ & $1.04 \mathrm{E}-04$ & $1.82 \mathrm{E}-02$ \\
\hline GO:0014068 & Positive regulation of phosphatidylinositol 3-kinase cascade & P & $4.51 \mathrm{E}-05$ & 9.49E-03 \\
\hline GO:0022603 & Regulation of anatomical structure morphogenesis & P & $2.20 \mathrm{E}-04$ & 2.89E-02 \\
\hline GO:0060828 & Regulation of canonical Wnt receptor signaling pathway & P & $4.33 \mathrm{E}-04$ & $4.82 \mathrm{E}-02$ \\
\hline GO:0042306 & Regulation of protein import into nucleus & P & $4.58 \mathrm{E}-04$ & 4.97E-02 \\
\hline GO:0035150 & Regulation of tube size & P & 1.79E-04 & 2.43E-02 \\
\hline GO:0009611 & Response to wounding & $P$ & $1.00 \mathrm{E}-04$ & 1.80E-02 \\
\hline GO:0048731 & System development & P & $5.66 \mathrm{E}-05$ & 1.17E-02 \\
\hline GO:0003018 & Vascular process in circulatory system & $P$ & $2.80 \mathrm{E}-05$ & $6.85 \mathrm{E}-03$ \\
\hline GO:0004872 & Receptor activity & $\mathrm{F}$ & 7.78E-06 & $2.65 \mathrm{E}-03$ \\
\hline GO:0015629 & Actin cytoskeleton & C & $1.44 \mathrm{E}-06$ & $6.80 \mathrm{E}-04$ \\
\hline GO:0009925 & Basal plasma membrane & C & $1.29 \mathrm{E}-04$ & $2.10 \mathrm{E}-02$ \\
\hline GO:0009986 & Cell surface & C & 2.67E-07 & $1.74 \mathrm{E}-04$ \\
\hline GO:0005911 & cell-cell junction & C & $1.54 \mathrm{E}-04$ & 2.30E-02 \\
\hline GO:0030139 & Endocytic vesicle & C & 1.69E-04 & 2.37E-02 \\
\hline GO:0005615 & Extracellular space & C & $1.15 \mathrm{E}-04$ & 1.95E-02 \\
\hline GO:0005887 & Integral to plasma membrane & C & $1.42 \mathrm{E}-04$ & 2.21E-02 \\
\hline GO:0043005 & Neuron projection & C & 7.12E-05 & $1.39 \mathrm{E}-02$ \\
\hline
\end{tabular}

Terms listed here are filtered for specificity and all are significant after a Benjamini-Hochberg correction (BH FDR). $\mathrm{P}=$ biological process, $\mathrm{C}=$ cellular component, and $\mathrm{F}=$ molecular function. 
The consistent signature of selection on genes associated with immunity, in conjunction with the latitudinal diversity gradient (LDG) and its logical corollary that increased biotic and host/parasite interactions are more prevalent in the tropics, led us to examine molecular differences between tropical and temperate rodents. For this we focused on developing immunogenetic resources for a group of non-model rodents that have representative species in both temperate and tropical latitudes. Previously, there have been cursory surveys of MHC diversity in the D. spectabilis genome [36]. However, genomic resources in this group are limited and those that do exist (e.g., a low-coverage genome in $D$. ordii [65]) are poorly annotated and too shallow for effective use in sequence assembly across the Heteromyidae [66]. Thus, we conducted RNA-sequencing to de novo assemble transcriptomes that we used to quantify gene expression and to test for divergent selection between the tropical and temperate species.

Our adult spleen transcriptome assemblies yielded a comparable number of contigs to our previous transcriptome work with these species [35] with slightly more in D. spectabilis (see Table 3). However, this increase in contig number is accompanied by a slightly shorter mean contig length due to a slightly more fragmented assembly. Regardless, we obtained an average of 24,594,286 reads per library $(12,297,143$ paired end reads per individual library) or $98,377,144$ reads per species $(49,188,572$ paired end reads per species). BLAST annotation identified a mean of 11,626 genes per species, roughly $63 \%$ of which were expressed across all three species at levels high enough to pass our filtering for the DE test. Although not significant after FDR correction, the identification of several immunity related GO terms as overrepresented in the overall $H$. desmarestianus spleen transcriptome are noteworthy. In particular, the presence of GO terms such as GO:0002215: defense response to nematode are of interest considering that Heteromys sp. are known to be infected by nematodes and a new species of Vexillata was previously identified in H. desmarestianus [67].

Given the mean library size, we utilized DESeq [51] to test for $\mathrm{DE}$ because it is one of the more conservative methods for detecting DE [68] and is consistent with other approaches such as edgeR [35,69]. 1,274 genes were significantly DE in each of two separate tests of the tropical $H$. desmarestianus versus $C$. baileyi and D. spectabilis. The
DE genes showed a relatively consistent pattern with only $11.8 \%$ of genes switching direction of DE between the two comparisons (i.e., $88.2 \%$ did not change direction). We encountered 632 genes that were consistently upregulated in $H$. desmarestianus and 492 genes that were consistently downregulated in $H$. desmarestianus. The consistent DE pattern for these genes in the tropical $H$. desmarestianus relative to the temperate species is of great interest and may indicate expression patterns shaped by environmental factors.

Compared to our previous work with kidney transcriptomes of these species, fewer genes were DE between the spleen transcriptomes of the three species $(1,274$ here compared to 1,897 in kidney). Interestingly, there was relatively little overlap in the genes that were DE in $H$. desmarestianus spleen and the genes that were DE in our previous kidney transcriptome work (313 genes that were $\mathrm{DE}$ in both kidney and spleen). This leaves some 811 genes that had a consistent pattern of DE in spleen tissue between $H$. desmarestianus and each of the temperate latitude species that does not appear to be due to constitutive organismal differences in gene expression between the taxa. Clearly more tissues and individuals are needed to confirm this result, but patterns of DE in these 811 genes may be due to immunity related or spleen specific differences.

When considering the ontologies associated with the 632 genes upregulated genes, we found statistical overrepresentation or underrepresentation of 126 GO terms relative to the rest of the genes tested for DE. This contrasts with the absence of overrepresentation or underrepresentation of GO terms within the set of genes that were downregulated in $H$. desmarestianus. The 126 GO terms overrepresented or underrepresented in the upregulated genes include several implicated in the vertebrate immune response (e.g., response to wounding, endocytic vesicle, etc.). Further, when DE genes are queried specifically for GO terms such as "innate immune response" and "antigen presentation", we find genes associated with innate and adaptive immune response, respectively. Specifically, 17 genes are annotated with "innate immune response" and 6 genes are annotated with "antigen presentation" in the set of 632 upregulated genes (see Additional file 1: Table S2 and S3). Overall, 31 of the 632 upregulated genes (4.9\%) were annotated with one or more GO terms that contained the phrase "immune response".

Table 5 Genes under positive selection according to the branch-site test

\begin{tabular}{|c|c|c|c|c|c|c|}
\hline $\begin{array}{l}\text { Swiss-Prot } \\
\text { symbol }\end{array}$ & Gene symbol & Protein name & $\begin{array}{l}\text { dn/ds branch-site } \\
\text { test }\end{array}$ & $\begin{array}{l}\text { Number } \\
\text { of sites }\end{array}$ & $\begin{array}{l}\text { P-value branch-site } \\
\text { test }\end{array}$ & $\begin{array}{c}P_{\text {adj }} \text { branch-site } \\
\text { test }\end{array}$ \\
\hline zdhc5 & Zdhhc5 & $\begin{array}{c}\text { Palmitoyltransferase ZDHHC5 } \\
\text { Ubiquitin-conjugating enzyme E2 N }\end{array}$ & 66.65 & 2145 & 0.0159 & 1.00 \\
\hline klf10 & KIf10 & Krueppel-like factor 10 & 46.30 & 1434 & 0.0291 & 1.00 \\
\hline ska1 & Skal & Spindle and kinetochore-associated protein 1 & 65.38 & 759 & 0.0115 & 0.91 \\
\hline
\end{tabular}

None were significant after FDR correction. 
It may be that the predicted pathogen load encountered by tropical species has resulted in elevated expression of specific immunity related genes or DE of specific types of immune response genes (e.g. different emphasis on innate vs. adaptive immunity relative to temperate species). However, the DE patterns that we observed could result from other sources such as developmental differences (though we minimized this by sampling only adults for all species) or temporal patterns of expression. Clearly, more research is necessary to confirm their consistent upregulation in $H$. desmarestianus spleen across additional variables (e.g., time points, body conditions, parasite load, etc.).

Of additional interest is the consideration of these expression results in addition to the results of our selection tests. Our initial reciprocal best blast analysis yielded roughly 1,150 genes (both partial and full cds) that were putative orthologues across all three species. Following the M. musculus EST analysis, length filtering, and subsequent MUSCLE alignment, we obtained sequence alignments of 395 genes to test for evidence of positive selection. This represents roughly $3 \%$ of the genes that we annotated in the $H$. desmarestianus adult spleen transcriptome. Within these 395 genes, 35 (8.9\% of genes tested for selection) were significantly DE, but none of these DE genes showed evidence of selection in our tests. We were able to detect departures from neutral models and detect cases where there was preliminary evidence of positive selection along the tropical $H$. desmarestianus branch (i.e., cases where the branch-sites test was significant before correction for multiple testing). We were mainly interested in those genes that not only showed departure from neutral models of sequence evolution, but those that also showed strong evidence of positive selection (elevated $\mathrm{dn} / \mathrm{ds}$ ) on the branch leading to $H$. desmarestianus. Such genes show divergence of the $H$. desmarestianus sequence from the other two species that served as temperate latitude environmental contrasts.

Automated sequence alignment can lead to an increased incidence of false positives $[70,71]$, so we required all of our putative orthologue sets to have fulllength coverage of known $M$. musculus protein coding sequence. We also screened all alignments that indicated elevated $\mathrm{dn} / \mathrm{ds}$ ratios along the $H$. desmarestianus branch and where the alternative model differed from the null initially $(\mathrm{p}<0.05, \mathrm{df}=1)$. During this latter step, we ultimately removed three alignments that were confounded by gaps, resulting in three genes with preliminary evidence of selection before correction for multiple testing. Our strict and conservative filtering methods may have lowered detection of positive selection to a point where the occurrence of false positives is unlikely even though the existence of false negatives is probable.
It is likely that the spleen transcriptome of $H$. desmarestianus contains additional genes that are under positive selection that were either removed from testing by our filtering, were not expressed at high enough levels to obtain full cds in all three species, could be heteromyid specific, or that could not be detected reliably using divergence based approaches. Ideally, further sequencing and another selection test with additional tropical and temperate heteromyids would be utilized to confirm the pattern observed herein and add statistical power to detect the extent of positive selection on the remaining 97\% of the $H$. desmarestianus spleen transcriptome.

The three genes with some evidence of selection represent only $0.8 \%$ of our filtered set of genes used for the selection tests (395 genes), which falls well below other estimates of selection from genomic scans of divergence (e.g. $6 \%$ in [72], $8 \%$ in [73], and $20 \%$ in [74]). A consequence of our conservative filtering was that we were left with genes that were expressed at high enough levels to completely assemble full transcripts for all three species. This probable bias to highly expressed genes could have contributed to the lower incidence of positive selection in our data compared to the studies cited above that looked at a majority of the genes in the genome. It has been suggested that genes that are expressed across a wide range of tissues tend to have a lower rate of nonsynonymous substitutions than genes with expression limited to one or a few tissues [75]. Indeed, when we compared the genes tested for selection here to those that were tested for selection in our previous study on the kidney transcriptomes of these species we found an overlap of 90 genes. The identification of these genes in additional tissues would be needed but these may be highly conserved genes that have expression across many organ systems and represent about $23 \%$ of the genes tested for selection herein.

A functional link between the gene and immunity is desirable in order to make the leap that these patterns of divergence between species are driven by parasite-(or pathogen) mediated selection in $H$. desmarestianus. There is no clear direct link between immune response and gene function for the genes listed in Table 5, and it is possible that the evolutionary pattern observed for these genes could be due to selective pressures other than immunity such as life history or habitat differences among these three heteromyids. For example, $H$. desmarestianus is native to wet areas, whereas the other two species inhabit xeric habitat $[20,35]$.

However, this lack of an obvious immune function does not preclude the possibility that selection is linked to immunity in some indirect way. Indeed, one textbook example of an immune related gene is selection on human $\beta$-globin. The top gene ontologies for this gene are heme binding, iron ion binding, oxygen binding, and 
oxygen transporter activity, and no immune function is apparent. However, we now know that the sickle cell allele for this gene is actively maintained in human populations due to the selective advantage of conferring malaria resistance $[63,76]$. Further taxonomic sampling may confirm the selective significance of the genes listed in Table 5; these may indeed function in the immune response, but mechanistic studies are needed to evaluate this possibility. Of course, many of the other genes that are present in all three species (including those that were DE) may be under selection due to a function in immune response in the tropical species but require characterization of full-length coding sequences to be tested further.

\section{Conclusions}

We have characterized the transcriptomes of three heteromyid rodents, identified a suite of genes that have been upregulated in $H$. desmarestianus, and identified several genes that exhibit preliminary evidence of positive selection. Their divergence from orthologues in temperate taxa could be driven by the selection pressure of elevated parasite abundance in tropical environments, be the result of other life history differences between these taxa, or be the result of stochastic processes. Further work is needed to combine direct estimates of sequence diversity for these and additional genes with estimates of parasite load and infection status in additional populations and species across a latitudinal gradient. The transcriptomes described herein would facilitate such an expanded study of sequence and expression differences between heteromyids from temperate and tropical latitudes. Additionally, these transcriptomes provide significant genetic resources from an immune tissue for members of all three of the heteromyid subfamilies.

\section{Availability of supporting data}

The data sets supporting the results of this article are available in the NCBI Short Read Archive and DataDryad repository (details below):

- Illumina sequence reads: NCBI SRA: Reads can be found with the entries for NCBI BioProjects: PRJNA261897, PRJNA261898, and PRJNA261902.

- Final transcriptome assemblies, list of contigs, putative blast annotation, and $\mathrm{R}$ and perl scripts are available on Dryad, http://dx.doi.org/10.5061/dryad.qn474

\section{Additional file}

Additional file 1: Table S1. List of GO terms from Fisher's exact test for gene enrichment in the spleen transcriptome of Heteromys desmarestianus relative to Dipodomys spectabilis and Chaetodipus baileyi. Terms that are 'over' enriched are overrepresented in $\mathrm{H}$. desmarestianus. FDR was $>.05$ for all terms so no terms were significant after Benjamini-Hochberg correction. Table S2 List of genes that were upregulated in Heteromys desmarestianus and annotated with the GO term: "innate immune response". Table S3 List of genes that were upregulated in Heteromys desmarestianus and annotated with any GO term containing the phrase "antigen presentation".

\section{Competing interests}

The authors declare that they have no competing interests.

\section{Authors' contributions}

NJM planned the study, collected samples, conducted RNA-seq, participated in bioinformatics analyses, and drafted the manuscript. JAD participated in the design of the project, supervised the project, and helped draft the manuscript. Both authors read and approved the final manuscript.

\section{Authors' information}

NJM was formerly a graduate student and postdoctoral researcher in the Department of Forestry \& Natural Resources at Purdue University. NJM is now a postdoctoral researcher in the Department of Population Medicine \& Diagnostic Sciences at Cornell University. JAD was NJM's advisor and is a Professor and University Faculty Scholar in the Department of Forestry \& Natural Resources and the Department of Biological Sciences at Purdue University.

\section{Acknowledgments}

This work was funded through a supplement to a National Science Foundation award (DEB-0816925), a NSF doctoral dissertation improvement grant to N.J.M, (DEB-1110421), and University Faculty Scholar funds provided to J.A.D.'s lab through Purdue's Office of the Provost. We thank two anonymous reviewers for helpful feedback and suggestions on an earlier version of this manuscript. We thank Andrea Romero for help trapping; Peter Waser for help in the field; Ruby Liu for help in the lab; Janna Willoughby for help with figures; Phillip San Miguel, Sam Martin, and Purdue University's Genomics Core Facility for help with cDNA sequencing; and Jyothi Thimmapuram of the Purdue Bioinformatics core for suggestions regarding joint assembly of 454 and Illumina data. Additionally, we are grateful for comments and feedback on this manuscript from members of the DeWoody Lab.

\section{Author details}

${ }^{1}$ Department of Population Medicine and Diagnostic Sciences, Cornell University, S3-111 Schurman Hall, Tower Road, Ithaca, NY 14853, USA. ${ }^{2}$ Department of Forestry and Natural Resources, Purdue University, West Lafayette, IN 47907, USA. ${ }^{3}$ Department of Biological Sciences, Purdue University, West Lafayette, IN 47907, USA.

Received: 27 June 2014 Accepted: 16 October 2014

Published: 24 October 2014

\section{References}

1. Cresko WA, Amores A, Wilson C, Murphy J, Currey M, Phillips P, Bell MA, Kimmel CB, Postlethwait JH: Parallel genetic basis for repeated evolution of armor loss in Alaskan threespine stickleback populations. Proc Natl Acad Sci U S A 2004, 101:6050-6055.

2. Shapiro MD, Marks ME, Peichel CL, Blackman BK, Nereng KS, Jónsson B, Schluter D, Kingsley DM: Genetic and developmental basis of evolutionary pelvic reduction in threespine sticklebacks. Nature 2004, 428:717-723.

3. Schemske DW, Mittelbach GG, Cornell HV, Sobel JM, Roy K: Is there a latitudinal gradient in the importance of biotic interactions? Annu Rev Ecol Evol Syst 2009, 40:245-269.

4. Dobzhansky T: Evolution in the tropics. Am Sci 1950, 38:209-221.

5. Janzen $\mathrm{DH}$ : Herbivores and the number of tree species in tropical forests. Am Nat 1970, 104:501-528.

6. Connell $\mathrm{JH}$ : On the role of natural enemies in preventing competitive exclusion in some marine animals and in rain forest trees. Dyn Popul 1971, 298:312.

7. Hochberg ME, van Baalen M: Antagonistic coevolution over productivity gradients. Am Nat 1998, 152:620-634. 
8. Ricklefs RE, Sheldon KS: Malaria prevalence and white-blood-cell response to infection in a tropical and in a temperate thrush. Auk 2007, 124:1254-1266.

9. Møller AP: Evidence of larger impact of parasites on hosts in the tropics: investment in immune function within and outside the tropics. Oikos 1998, 82:265-270.

10. Owen-Ashley NT, Hasselquist D, Råberg L, Wingfield JC: Latitudinal variation of immune defense and sickness behavior in the whitecrowned sparrow (Zonotrichia leucophrys). Brain Behav Immun 2008, 22:614-625.

11. Møller AP, Martín-Vivaldi M, Merino S, Soler JJ: Density-dependent and geographical variation in bird immune response. Oikos 2006, 115:463-474

12. Ricklefs RE: Embryonic development period and the prevalence of avian blood parasites. Proc Natl Acad Sci U S A 1992, 89:4722-4725.

13. Salkeld DJ, Trivedi M, Schwarzkopf L: Parasite loads are higher in the tropics: temperate to tropical variation in a single host-parasite system. Ecography (Cop) 2008, 31:538-544.

14. Nunn $C L$, Altizer SM, Sechrest W, Cunningham AA: Latitudinal gradients of parasite species richness in primates. Divers Distrib 2005, 11:249-256.

15. Guernier V, Hochberg ME, Guégan J-F: Ecology drives the worldwide distribution of human diseases. PLOS Biol 2004, 2:e141.

16. Cumming GS: Using habitat models to map diversity: pan-African species richness of ticks (Acari: Ixodida). J Biogeogr 2000, 27:425-440.

17. Kolaczkowski B, Kern AD, Holloway AK, Begun DJ: Genomic differentiation between temperate and tropical Australian populations of Drosophila melanogaster. Genetics 2011, 187:245-260.

18. Dionne M, Miller KM, Dodson JJ, Caron F, Bernatchez L: Clinal variation in MHC diversity with temperature: evidence for the role of host-pathogen interaction on local adaptation in Atlantic salmon. Evolution 2007, 61:2154-2164

19. Alexander LF, Riddle BR: Phylogenetics of the New World rodent family Heteromyidae. J Mammal 2005, 86:366-379.

20. Genoways HH, Brown JH (Eds): Biology of the Heteromyidae. Provo, Utah: American Society of Mammalogists; 1993.

21. Hafner JC, Light JE, Hafner DJ, Hafner MS, Reddington E, Rogers DS, Riddle BR: Basal clades and molecular systematics of heteromyid rodents. J Mammal 2007, 88:1129-1145.

22. Anderson RP: Taxonomy, distribution, and natural history of the genus Heteromys (Rodentia : Heteromyidae) in western Venezuela, with the description of a dwarf species from the Peninsula de Paraguana. Am Museum Novit 2003, 3396:1-43.

23. Rogers DS: Genic evolution, historical biogeography, and systematic relationships among spiny pocket mice (subfamily Heteromyinae). J Mammal 1990, 71:668-685.

24. IUCN Red List of Threatened Species. [www.iucnredlist.org]

25. Olson DM, Dinerstein E, Wikramanayake ED, Burgess ND, Powell GVN, Underwood EC, D'amico JA, Itoua I, Strand HE, Morrison JC, Loucks CJ, Allnutt TF, Ricketts TH, Kura Y, Lamoreux JF, Wettengel WW, Hedao P, Kassem KR: Terrestrial ecoregions of the world: A new map of life on Earth. Bioscience 2001, 51:933-938.

26. Light JE, Hafner MS: Codivergence in heteromyid rodents (Rodentia: Heteromyidae) and their sucking lice of the genus Fahrenholzia (Phthiraptera: Anoplura). Syst Bol 2008, 57:449-465.

27. Best TL: Dipodomys spectabilis. Mamm Species 1988, 311:1-10.

28. Vorhies $\subset$, Taylor WP: Life history of the kangaroo rat, Dipodomys spectabilis spectabilis Merriam. United States Dep Agric Tech Bull 1922, 1091:1-40.

29. Eisenberg JF: The Behavior of Heteromyid Rodents. Berkeley, California: University of California Publications in Zoology; 1963:111.

30. Steinwald MC, Swanson BJ, Doyle JM, Waser PM: Female mobility and the mating system of the banner-tailed kangaroo rat (Dipodomys spectabilis). J Mammal 2013, 94:1258-1265.

31. Howell AB, Gersh I: Conservation of water by the rodent Dipodomys. J Mammal 1935, 16:1-9.

32. Vimtrup B, Schmidt-Nielsen B: The histology of the kidney of kangaroo rats. Anat Rec 1952, 114:515-528.

33. Urity VB, Issaian T, Braun EJ, Dantzler WH, Pannabecker TL: Architecture of kangaroo rat inner medulla: segmentation of descending thin limb of Henle's loop. Am J Physiol Regul Integr Comp Physiol 2012, 302:R720-R726.

34. Issaian T, Urity VB, Dantzler WH, Pannabecker TL: Architecture of vasa recta in the renal inner medulla of the desert rodent Dipodomys merriami: potential impact on the urine concentrating mechanism. Am J Physiol Regul Integr Comp Physiol 2012, 303:R748-R756.
35. Marra NJ, Romero A, DeWoody JA: Natural selection and the genetic basis of osmoregulation in Heteromyid rodents as revealed by RNA-seq. Mol Ecol 2014, 23:2699-2711.

36. Busch JD, Waser PM, DeWoody JA: Characterization of expressed class II $\mathrm{MHC}$ sequences in the banner-tailed kangaroo rat (Dipodomys spectabilis) reveals multiple DRB loci. Immunogenetics 2008, 60:677-688.

37. Del Portillo HA, Ferrer M, Brugat T, Martin-Jaular L, Langhorne J, Lacerda MVG: The role of the spleen in malaria. Cell Microbiol 2012, 14:343-355.

38. Mebius RE, Kraal G: Structure and function of the spleen. Nat Rev Immunol 2005, 5:606-616.

39. Brendolan A, Rosado MM, Carsetti R, Selleri L, Dear TN: Development and function of the mammalian spleen. BioEssays 2007, 29:166-177.

40. Den Haan JMM, Kraal G: Innate immune functions of macrophage subpopulations in the spleen. J Innate Immun 2012, 4:437-445.

41. Titus RG: Salivary gland lysate from the sand fly Lutzomyia longipalpis suppresses the immune response of mice to sheep red blood cells in vivo and concanavalin A in vitro. Exp Parasitol 1998, 89:133-136.

42. Cross ML, Cupp MS, Cupp EW, Galloway AL, Enriquez FJ: Modulation of murine immunological responses by salivary gland extract of Simulium vittatum (Diptera: Simuliidae). J Med Entomol 1993, 30:928-935.

43. Marra NJ, Eo SH, Hale MC, Waser PM, DeWoody JA: A priori and a posteriori approaches for finding genes of evolutionary interest in non-model species: osmoregulatory genes in the kidney transcriptome of the desert rodent Dipodomys spectabilis (banner-tailed kangaroo rat). Comp Biochem Physiol Part D Genomics Proteomics 2012, 7:328-339.

44. Zhu YY, Machleder EM, Chenchik A, Li R, Siebert PD: Reverse transcriptase template switching: A SMART (TM) approach for full-length cDNA library construction. Biotechniques 2001, 30:892-897.

45. Hale MC, McCormick CR, Jackson JR, Dewoody JA: Next-generation pyrosequencing of gonad transcriptomes in the polyploid lake sturgeon (Acipenser fulvescens): the relative merits of normalization and rarefaction in gene discovery. BMC Genomics 2009, 10:203.

46. Eo SH, Doyle JM, Hale MC, Marra NJ, Ruhl JD, Dewoody JA: Comparative transcriptomics and gene expression in larval tiger salamander (Ambystoma tigrinum) gill and lung tissues as revealed by pyrosequencing. Gene 2012, 492:329-338

47. Schmieder R, Edwards R: Fast identification and removal of sequence contamination from genomic and metagenomic datasets. PLOS ONE 2011, 6:e17288.

48. Grabherr MG, Haas BJ, Yassour M, Levin JZ, Thompson DA, Amit I, Adiconis X, Fan L, Raychowdhury R, Zeng Q, Chen Z, Mauceli E, Hacohen N, Gnirke A, Rhind N, di Palma F, Birren BW, Nusbaum C, Lindblad-Toh K, Friedman N, Regev A: Full-length transcriptome assembly from RNA-Seq data without a reference genome. Nat Biotechnol 2011, 29:644-652.

49. Langmead B, Trapnell C, Pop M, Salzberg SL: Ultrafast and memoryefficient alignment of short DNA sequences to the human genome. Genome Biol 2009, 10:R25.

50. Li B, Dewey CN: RSEM: accurate transcript quantification from RNA-Seq data with or without a reference genome. BMC Bioinformatics 2011, $12: 323$

51. Anders S, Huber W: Differential expression analysis for sequence count data. Genome Biol 2010, 11:R106.

52. Edgar RC: MUSCLE: multiple sequence alignment with high accuracy and high throughput. Nucleic Acids Res 2004, 32:1792-1797.

53. Yang Z: PAML: a program package for phylogenetic analysis by maximum likelihood. Comput Appl Biosci 1997, 13:555-556.

54. Yang Z: PAML 4: phylogenetic analysis by maximum likelihood. $\mathrm{Mol}$ Biol Evol 2007, 24:1586-1591.

55. Altschul SF, Gish W, Miller W, Myers EW, Lipman DJ: Basic local alignment search tool. J Mol Biol 1990, 215:403-410.

56. Götz S, Garcia-Gomez JM, Terol J, Williams TD, Nagaraj SH, Nueda MJ, Robles M, Talon M, Dopazo J, Conesa A: High-throughput functional annotation and data mining with the Blast2GO suite. Nucleic Acids Res 2008, 36:3420-3435

57. Li B, Ruotti V, Stewart RM, Thomson JA, Dewey CN: RNA-Seq gene expression estimation with read mapping uncertainty. Bioinformatics 2010, 26:493-500.

58. Benjamini $Y$, Hochberg $Y$ : Controlling the false discovery rate: a practical and powerful approach to multiple testing. J R Stat Soc Ser B 1995, 57:289-300.

59. Yang Z, Nielsen R: Synonymous and nonsynonymous rate variation in nuclear genes of mammals. J Mol Evol 1998, 46:409-418. 
60. Yang Z: Likelihood ratio tests for detecting positive selection and application to primate lysozyme evolution. Mol Biol Evol 1998, 15:568-573.

61. Zhang J, Nielsen R, Yang Z: Evaluation of an improved branch-site likelihood method for detecting positive selection at the molecular level. Mol Biol Evol 2005, 22:2472-2479.

62. Yang Z, Wong WSW, Nielsen R: Bayes empirical bayes inference of amino acid sites under positive selection. Mol Biol Evol 2005, 22:1107-1118.

63. Barreiro LB, Quintana-Murci L: From evolutionary genetics to human immunology: how selection shapes host defence genes. Nat Rev Genet 2010, 11:17-30.

64. Sabeti PC, Schaffner SF, Fry B, Lohmueller J, Varilly P, Shamovsky O, Palma A Mikkelsen TS, Altshuler D, Lander ES: Positive natural selection in the human lineage. Science 2006, 312:1614-1620.

65. Lindblad-Toh K, Garber M, Zuk O, Lin MF, Parker BJ, Washietl S, Kheradpour P, Ernst J, Jordan G, Mauceli E, Ward LD, Lowe CB, Holloway AK, Clamp M, Gnerre S, Alföldi J, Beal K, Chang J, Clawson H, Cuff J, Di Palma F, Fitzgerald S, Flicek P, Guttman M, Hubisz MJ, Jaffe DB, Jungreis I, Kent WJ, Kostka D, Lara M, et al: A high-resolution map of human evolutionary constraint using 29 mammals. Nature 2011, 478:476-482.

66. DeWoody JA, Abts KC, Fahey AL, Ji Y, Kimble SJA, Marra NJ, Wijayawardena BK, Willoughby JR: Of contigs and quagmires: next-generation sequencing pitfalls associated with transcriptomic studies. Mol Ecol Resour 2013, 13:551-558.

67. Falcón-Ordaz J, García-Prieto L: A new species of Vexillata (Nematoda: Trichostrongylina: Ornithostrongylidae) parasite of Heteromys desmarestianus (Rodentia: Heteromyidae) from Costa Rica. J Parasitol 2005, 91:329-334.

68. Soneson C, Delorenzi M: A comparison of methods for differential expression analysis of RNA-seq data. BMC Bioinformatics 2013, 14:91.

69. Robinson MD, McCarthy DJ, Smyth GK: edgeR: a Bioconductor package for differential expression analysis of digital gene expression data. Bioinformatics 2010, 26:139-140.

70. Fletcher $W$, Yang $Z$ : The effect of insertions, deletions, and alignment errors on the branch-site test of positive selection. Mol Biol Evol 2010, 27:2257-2267.

71. Markova-Raina P, Petrov D: High sensitivity to aligner and high rate of false positives in the estimates of positive selection in the 12 Drosophila genomes. Genome Res 2011, 21:863-874.

72. Brieuc MSO, Naish KA: Detecting signatures of positive selection in partial sequences generated on a large scale: pitfalls, procedures and resources. Mol Ecol Resour 2011, 11(Suppl 1):172-183.

73. Anisimova M, Bielawski J, Dunn K, Yang Z: Phylogenomic analysis of natural selection pressure in Streptococcus genomes. BMC Evol Biol 2007, 7:154

74. Clark AG, Glanowski S, Nielsen R, Thomas PD, Kejariwal A, Todd MA, Tanenbaum DM, Civello D, Lu F, Murphy B, Ferriera S, Wang G, Zheng X White TJ, Sninsky JJ, Adams MD, Cargill M: Inferring nonneutral evolution from human-chimp-mouse orthologous gene trios. Science 2003 , 302:1960-1963.

75. Duret L, Mouchiroud D: Determinants of substitution rates in mammalian genes: expression pattern affects selection intensity but not mutation rate. Mol Biol Evol 2000, 17:68-74

76. Allison AC: Protection afforded by sickle-cell trait against subtertian malareal infection. Br Med J 1954, 1:290-294.

doi:10.1186/1471-2164-15-929

Cite this article as: Marra and DeWoody: Transcriptomic characterization of the immunogenetic repertoires of heteromyid rodents. BMC Genomics 2014 15:929.

\section{Submit your next manuscript to BioMed Central and take full advantage of:}

- Convenient online submission

- Thorough peer review

- No space constraints or color figure charges

- Immediate publication on acceptance

- Inclusion in PubMed, CAS, Scopus and Google Scholar

- Research which is freely available for redistribution 